\title{
A real-time wearable emotion detection headband based on EEG measurement
}

\author{
Yue Wu, Yang Wei and John Tudor \\ Electronics and Computer Science \\ University of Southampton \\ SO17 1BJ, Southampton, UK
}

\begin{abstract}
:
A real-time emotion detection system based on electroencephalogram (EEG) measurement has been realised by means of an emotion detection headband coupled with printed signal acquisition electrodes and open source signal processing software (OpenViBE). Positive and negative emotions are the states classified and the Theta, Alpha, Beta and Gamma frequency bands are selected for the signal processing. It is found that, by using a combination of Power Spectral Density (PSD), Signal Power (SP) and Common Spatial Pattern (CSP) as the features, the highest subject-dependent accuracy $(86.83 \%)$ and independent accuracy $(64.73 \%)$ is achieved, when using Linear Discrimination Analysis (LDA) as the classification algorithm. The standard deviation of the results is 5.03. The electrode locations were then improved for the detection of emotion, by moving them from F1, F2, T3 and T4 to A1, F2, F7 and F8. The subject-dependent accuracy, using the improved locations, increased to $91.75 \%$ from $86.83 \%$ and $75 \%$ of participants achieved a classification accuracy higher than $90 \%$, compared with only $16 \%$ of participants before improving the electrode arrangement.
\end{abstract}

\section{Introduction}

Emotion detection is an emerging topic and emotions influence much of daily life such as reasoning and attention [1], decision making [2], well-being [3] and the quality of life [4]. The immune system is also related to the emotions as a weaker immune response is generally induced by negative emotions [5]. People who experience positive emotions have a lower risk of suffering from disease than those with negative emotions [6]. In addition, people with positive emotions frequently live longer and healthier lives than those with negative emotions [7]. Emotion detection offers the potential to identify negative emotions and address the causal factors, potentially leading to more positive emotions.

In the past, emotion was detected from the facial expression or tone of voice; however, the accuracy cannot be guaranteed because of misleading tonal changes or spurious facial expressions [8]. Alternatively, physiological signals are used such as skin temperature, the Electrocardiogram and the Electroencephalogram (EEG), which records the electrical brain activity on the scalp [9]. EEG is the most direct method because it measures the voltage changes caused by ionic current flows within the neurons of the brain which result from emotions [10]. The left hemisphere is more active during positive emotions and the right hemisphere during negative emotions [11]. This is because the alpha wave of the right hemisphere decreases with negative emotions and that of the left hemisphere decreases with positive emotions [12]. Such brain asymmetry can be applied for emotion detection based on EEG signals.

The primary objectives of EEG-based emotion detection research are to implement real-time measurement with high accuracy [9]. Previous research has identified important six major factors which affect the outcome [9]:

- Participant: The method for building emotion classification can be divided into subjectindependent and subject-dependent models. The former means that the classification model is built for the entire database, while the latter indicates the classification model is built for 
every new user [13]. The former is more difficult to achieve with high accuracy than the latter because of inter participant variability [14].

- Model of Emotion: The common dimensional model consists of two main dimensions which are valence and arousal [9]. The arousal emotion ranges from calm to excited and the valence emotion ranges from negative to positive [15]. This model is called the circumplex model [16] and is shown in Figure 1. It is the most widely used model, allowing emotions to be illustrated graphically and mapped on a coordinate system.

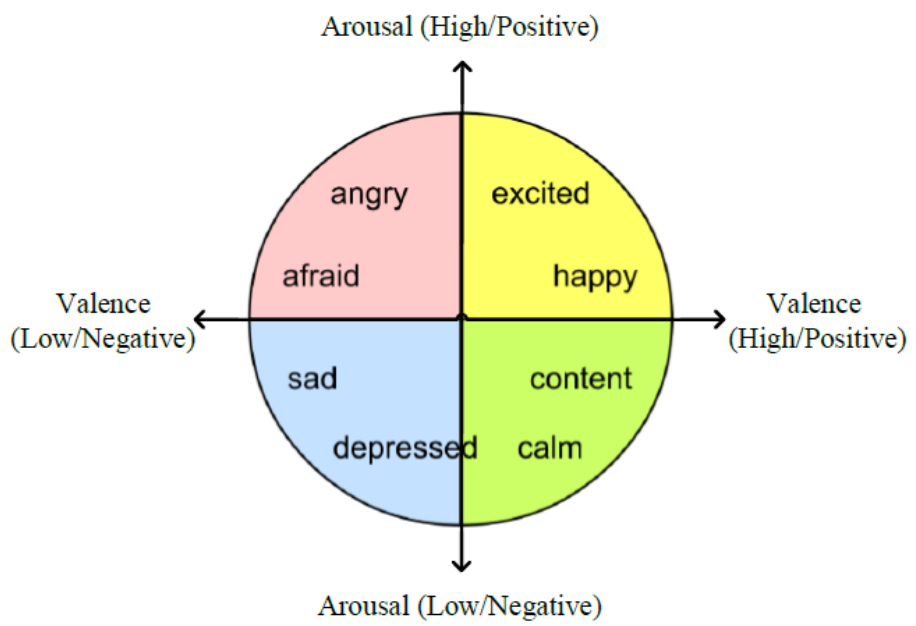

Figure 1. Arousal-valence model [16].

- Stimuli: External stimuli such as pictures, sounds and videos are commonly used to induce emotions in the participants.

- Features: Potential signal features are Power Spectral Density (PSD), Asymmetric Spatial Pattern (ASP), Spectral Power Asymmetry (ASM) and Asymmetric Spatial Pattern (ASP) [9].

- Temporal Window: The type of emotion targeted and the physiological signal determines the appropriate length of the temporal window for emotion detection which is typically from 0.5 to $4 \mathrm{~s}$ [17]. Our system is called real-time since it can provide a continuous display of emotions as they occur. However, we note there is a very short delay $(<1 \mathrm{~s})$ between, when the emotion occurs, and its display.

- Classifier: This factor determines the power consumption and the accuracy of the result. There are several machine learning algorithms which can be used as emotion classifiers such as Naive Bayes (NB) [18] and Support Vector Machine (SVM) [9]. In addition to the classifier, there is a Library for Support Vector Machines (LIBSVM) [18] which supports various SVM formulations for classification, regression, and distribution estimation.

The electrical activity recorded on the scalp are decomposed into five frequency bands, which are: delta $(1-3 \mathrm{~Hz})$, theta $(4-7 \mathrm{~Hz})$, alpha $(8-13 \mathrm{~Hz})$, beta $(14-30 \mathrm{~Hz})$, and gamma $(31-50 \mathrm{~Hz})$ [19]. In order to acquire brain activity, the 10-20 system of electrode placement, shown in Figure 2 [20], is used. This is an international standard of scalp electrode locations with each position identified by a name. 

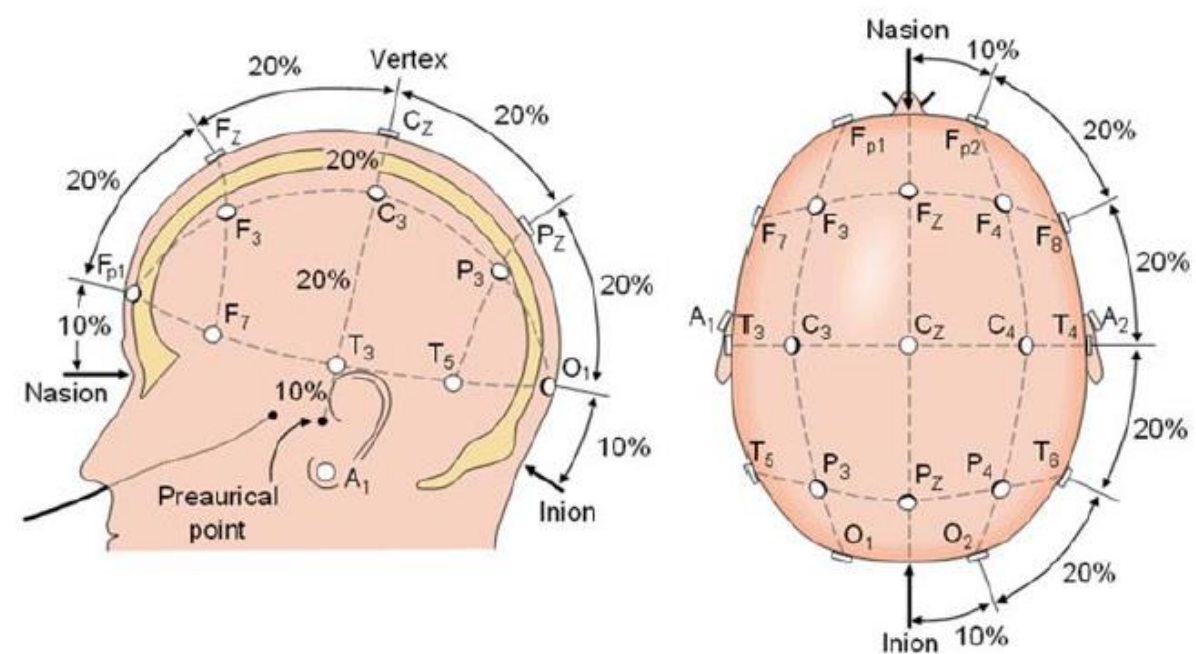

Figure 2. International 10-20 system seen from left (Left) and above (Right) [20].

Liu et al developed a real-time emotion detection system based on measuring EEG signals [21]. A Fractal Dimension algorithm was used and both arousal and valence were classified. Three channels were used with electrodes at the FC6, F4 and AF3 positions according to the 10-20 international standard [20]. An Emotiv headset [22] was implemented as the signal acquisition device and 12 participants were used to test the system. However, instead of using a standard classifier, Fractal Dimension (FD) values from a Higuchi Fractal Dimension (HFD) algorithm were compared with a predefined threshold. It is not clear as to how the threshold was defined and the accuracy of the emotion detection system was not specified [23].

Anh et al demonstrated a real-time emotion detection system [23]. The channels used were the same as reported in [21]: FC6, F4 and AF3. Fractal Dimension values as well as AV (arousal and valence) values were used as the features while SVM was used as the classifier [23] to classify five emotions: happy, sad, relaxed, neutral and angry. The EEG signal was collected using a Emotiv headset and a SAM (Self-Assessment Manikin) was implemented together with the circumplex model for emotion evaluation. The average subject-dependent classification accuracy was $70.5 \%$ from 20 participants. The experimental results indicated that the model in [23] successfully achieved accurate subject dependent detection but could only achieve a subject independent detection accuracy of $<10 \%$.

Jatupaiboon et al used the Support Vector Machine algorithm as the classifier for two channels to implement a real-time EEG-based happiness detection system using an Emotiv headset as the signal acquisition device [9]. The Power Spectral Density feature from the alpha, beta, delta, theta and gamma frequency bands was used. The temporal window was chosen as $1 \mathrm{~s}$ and the system classified the emotional state every $5 \mathrm{~s}$. Pictures and music were used as the stimuli to induce emotions. 10 participants were used to test the real-time system. The average accuracies of subject-independent and subject-dependent models were $65.12 \%$ and $75.62 \%$ respectively.

Matiko et al implemented another real-time emotion detection system in which the active electrodes were embedded within a headband and the data wirelessly transferred to a computer [24]. This design used flexible solar cells on the headband as a power supply and embedded the EEG signal processing electronics within the headband. The correlation coefficient between the signals from the printed active electrodes used and commercial passive electrodes was $70.88 \%$. The highest average subjectindependent classification accuracy achieved was $62.62 \%$ and the subject-dependent accuracy was 90 $\%$ [24]. The two channel EEG signals were obtained from the asymmetrical locations of AF3 and AF4. The Signal Power and the Oscillation features of the alpha band from two channels were also used for emotion detection classified by a Fuzzy Logic algorithm. 
This paper presents a real-time emotion detection system based on EEG signal measurement. Positive and negative emotions are the states classified. The signal is acquired using screen printed active dry electrodes which are assembled in a headband. The printing approach enables the fabric headband and electrodes to be customised in size to be suitable for a wide range of wearers. Active dry electrodes were selected because they reduce the noise and electrode impedance, compared with passive dry electrodes, thereby increasing signal quality. The signal is then processed by OpenViBE software to extract the features and classify emotions [25]. A novel algorithm, combining PSD, SP and CSP features, achieved the highest subject-dependent accuracy $(86.83 \%)$ and subject-independent accuracy $(64.73 \%)$ when using Linear Discrimination Analysis (LDA) as the classification algorithm. Subsequently improved electrode locations, at A1, F2, F7 and F8, increased the subject-dependent accuracy to $91.75 \%$ from $86.83 \%$, which is the highest compared with other reported similar approaches. The use of the open-source OpenVibe software and standard interface hardware, allows replication by other researchers of the signal processing and classification algorithm developed in this paper and we will release the source code into the public domain after publishing this paper. In summary this paper presents a novel combination of extraction features (PSD, SP and CSP) together with an improved electrode arrangement which avoids hairs. This is implemented on a printed headband offering headband size adjustment and the system uses open source software to achieve the highest reported subject dependent/independent accuracies (91.75\%/66.74\%) in real-time.

In this paper, section 2 presents a system overview followed by a description of the emotion detection headband, the EEG amplifier and microcontroller as well as the OpenViBE signal processing software. Section 3 provides the results together with a discussion starting with a performance analysis of the printed electrodes, the implementation of real-time emotion detection and the performance of a new electrode arrangement. Finally, section 4 provides conclusions.

\section{System design}

\subsection{System overview}

The emotion detection system consists of three parts, shown in Figure 3:

(1) The EEG measurement front end which is a headband with integrated printed active electrodes to acquire and pre-amplify the raw EEG signals;

(2) A biofeedback board called an "EEG-SMT" supplied by Olimex [26]. This is used to further amplify and digitize the acquired EEG signal;

(3) OpenViBE software which is used to extract features and classify emotions.

In summary, the printed electrodes are used to acquire the EEG signals from the human brain which are then amplified by two analogue amplifiers before digitisation by an analogue to digital convertor in a microcontroller. Finally, the data is transmitted to a computer through wires and processed by the OpenViBE software. Figure 3 presents these system components.

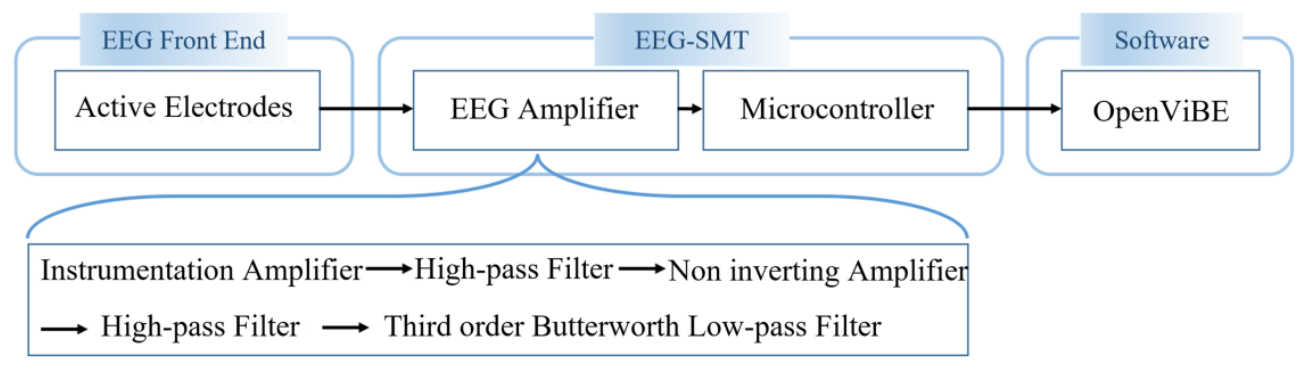

Figure 3. Block diagram of emotion detection system based on EEG. 


\subsection{EEG Front End}

The design of the EEG electrodes used in this paper is similar to the active electrodes described by Paul et al [27]. The active electrodes were printed on one side of a woven textile to allow wearability and breathability of the resulting garment [27]. The design is shown in Figure 4, which includes a conductive layer attached to a buffer amplifier.
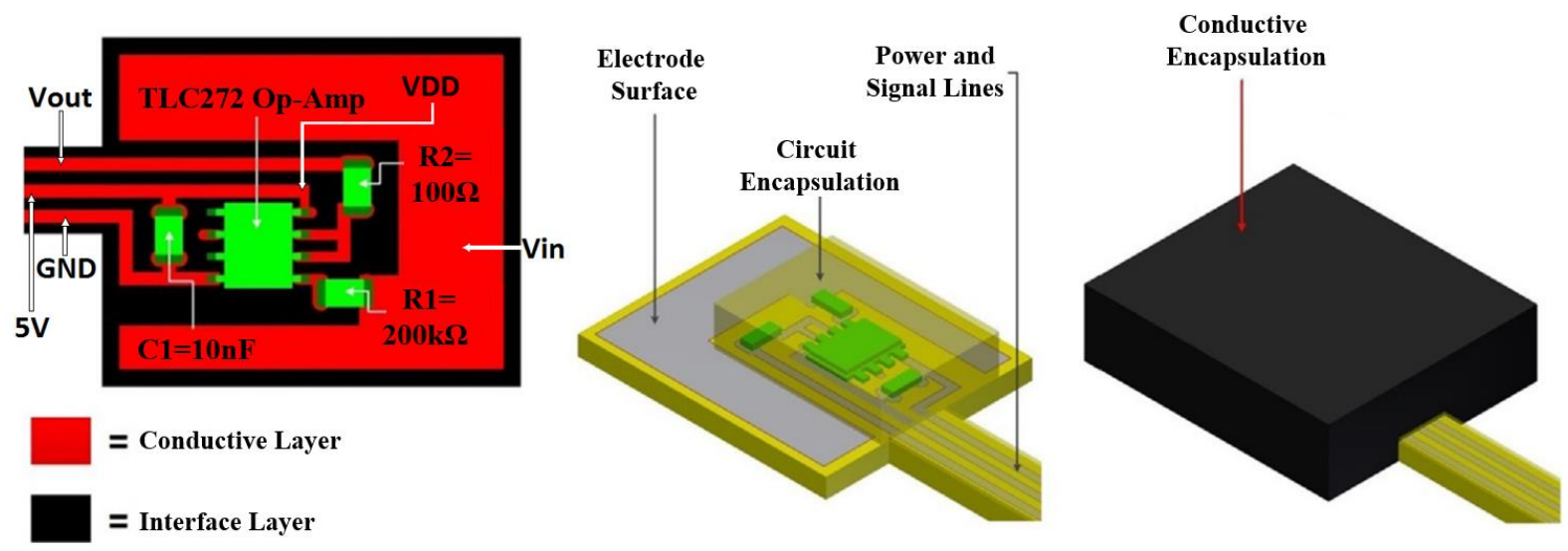

Figure 4. The design of screen printed active electrode [26].

The red area indicates the conductive layer and the black area on the left picture represents a printed interface layer on the textile under the conductive layer. After printing, electronic components (a 10 $\mathrm{nF}$ capacitor, a $100 \Omega$ resistor, a $200 \mathrm{k} \Omega$ resistor and a TLC 727 operational amplifier) were attached to the printed circuit using silver loaded epoxy adhesive. The circuit was then encapsulated by stencil printing a dielectric ink which is used to isolate the buffer amplifier circuit from the conductive encapsulation. Finally, the entire electrode was encapsulated by stencil printing carbon-loaded rubber which connects the electrodes to the skin. The encapsulation is shown in Figure 4 (middle and right), and the design and printing results of a single electrode is shown in Figure 5.

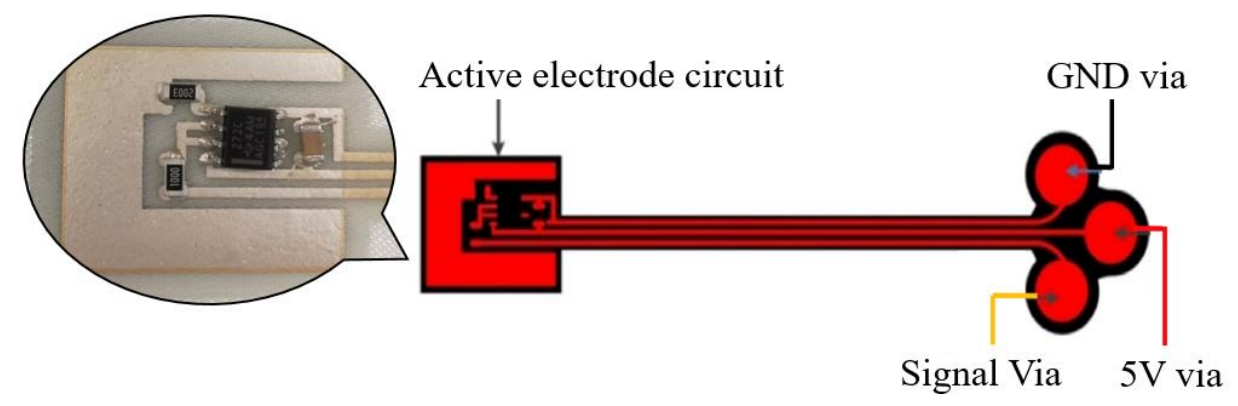

Figure 5. A photograph of printed electrode circuit before encapsulation (Left); A drawing of a single EEG electrode unit (Right).

Each electrode unit consists of three $9 \mathrm{~mm}$ diameter fabric snap connectors which connect to a $5 \mathrm{~V}$ external power supply and the amplifier on the EEG-SMT. The completed headband structure is shown in Figure 6. 

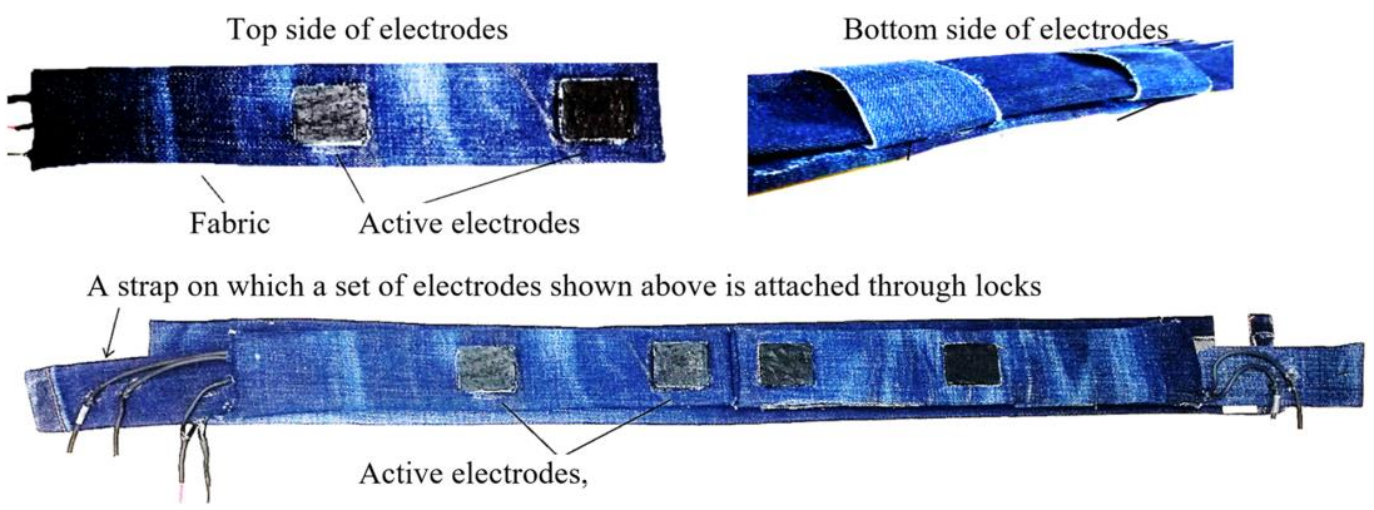

Figure 6. Top and bottom side of printed electrodes (Top). Overview of the headband (Bottom).

The four printed electrodes in the headband are used to acquire EEG signals from the locations of Fp1, Fp2, T3 and T4. Fp1, T3 (left hemisphere group) and Fp2, T4 (right hemisphere group) are selected to acquire signals from two different hemispheres of the brain. The default distance between two electrodes is $10.16 \mathrm{~cm}$ which is based on the international 10-20 electrode placement system [20]. Since different participants have different head perimeters, the distance between electrodes can be changed by moving them on the strap

\subsection{EEG-SMT}

As the raw EEG signals are pre-amplified within the printed electrodes, the signals from the headband are in the range $0.02 \mathrm{~V}$ to $0.2 \mathrm{~V}$; these signals therefore need to be further amplified to a suitable level for digitization and processing. This is achieved in the EEG-SMT board, shown in Figure 7. EEGSMT is the manufacturer's name for a bio-feedback board from Olimex [26] which contains both an analogue amplifier and a microcontroller. Both the software and hardware design of this board are fully open source and it has a compact size of $80 \mathrm{~mm} \times 42 \mathrm{~mm}$. The EEG-SMT is powered from a USB cable [26].

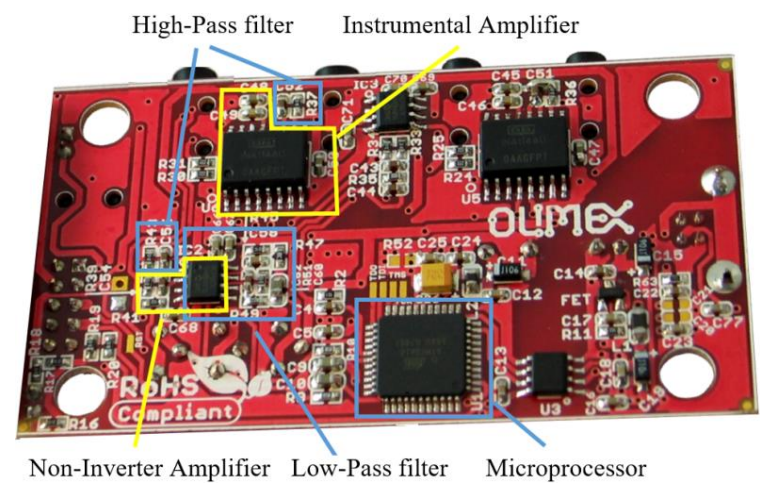

Figure 7. EEG-SMT board [26].

The EEG-SMT performs the following functions. An instrumentation amplifier INA114 [28] from Texas Instruments is used to attenuate the power line noise by means of its high common mode rejection ratio. A passive high-pass filter is then used to filter out any dc offset to avoid saturating the following stages by cutting off frequencies below $0.16 \mathrm{~Hz}$. After that, a non-inverter amplifier MCP607I/SN [29] from Microchip provides a gain of 40 and this is followed by another high pass filter to remove any dc offset. A third order Butterworth low pass filter with a cut-off frequency of 59 $\mathrm{Hz}$ is then used to reduce anti-aliasing effects caused by analogue to digital conversion. $59 \mathrm{~Hz}$ is the default value set by the EEG-SMT board and cannot be changed. The microprocessor, an ATmega16 from Atmel [30], is used to digitise the signals before transmission to a computer. 


\subsection{Software}

The OpenViBE software uses an open-source graphical programming language and can be used to design and test brain-computer interfaces [25]. It can therefore be used to acquire, filter, extract, classify and visualize brain signals in real-time. The three stages of the emotion detection flow using OpenViBE are:

Stage 1: Data Acquisition: The EEG signals are acquired by the printed electrodes and transmitted to the computer via the EEG-SMT. For the training of the classifier, a predefined stimulus label, either positive or negative, depending on the type of stimulus, is added to the acquired EEG signal. The recorded signal is then ready to be used in stage 2 .

Stage 2: Feature extraction: CSP, signal power and PSD are extracted from the EEG signal as the features of the signal to be classified.

Stage 3: Classifier training and Real-time emotion detection: The Linear Discriminant Analysis (LDA) classifier algorithm is initially trained using the labels from stage 1 and the features obtained from stage 2 to allow the classifier to distinguish different emotional classes. Once the training is completed the trained classifier can then be used to detect emotion in real-time.

\section{System Testing}

\subsection{Experimental rationale}

The performance of the system was evaluated by means of three experiments. The first evaluates the performance of the printed electrodes. The second builds the software system through real-time emotion detection experiments to select the most appropriate frequency band in which to extract the features. The third evaluates the current setup to further improve the accuracy.

\subsection{Experiment 1: Electrode testing}

Resistance and the correlation coefficient, compared with commercial electrodes, are used to assess the performance of the printed electrodes.

\subsubsection{Electrode resistance}

The resistance of the printed electrodes was measured using a Fluke 178 multimeter. Table 1 shows the average resistance of the printed electrodes when they were fabricated, after 30 days and after subsequent cleaning in IPA.

Table 1. The change of electrode resistance.

\begin{tabular}{ccc}
\hline Electrode resistance after: \\
\hline Fabricated & $\mathbf{3 0}$ days & $\begin{array}{c}\text { Cleaned by IPA } \\
\text { after } 30 \text { days }\end{array}$ \\
\hline$\sim 0.25 \Omega / \mathrm{cm}$ & $\sim 500 \Omega / \mathrm{cm}$ & $50-100 \Omega / \mathrm{cm}$ \\
\hline
\end{tabular}

The surface of the printed electrode becomes contaminated during wearing and contamination; dirt and grease can be observed on a lint free wipe after cleaning. The resistance reduced from $500 \Omega / \mathrm{cm}$ to $50-100 \Omega / \mathrm{cm}$ after cleaning the surface with a lint free wipe soaked in IPA. However, this is still above the resistance when first fabricated and it is believed that this irreversible change of resistance is caused by mechanical failure between the rubber and conductive layer after use, which reduces the adhesion of the rubber layer. This resistance increase will therefore reduce the signal magnitude and consequently the performance of the system. 


\subsubsection{Correlation coefficient of the electrodes}

All the printed electrodes were tested individually by measuring the EEG signal at the Fp1 position compared with standard commercial passive silver chloride electrodes of $1 \mathrm{~cm}$ diameter supplied by $3 \mathrm{M}$. The commercial electrodes and printed electrodes were placed side-by-side to record the EEG signals simultaneously for 3 minutes. A sample of the EEG signal measured using the printed and commercial electrodes during a $5 \mathrm{~s}$ period is shown in Figure 8.

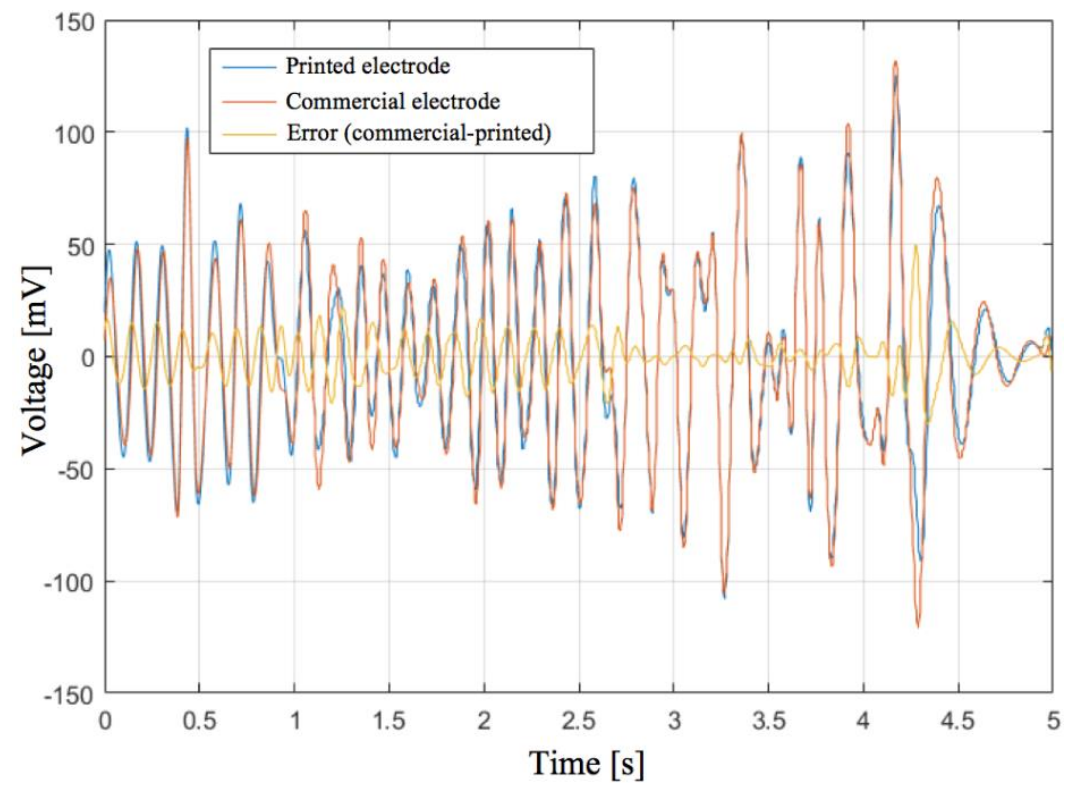

Figure 8. EEG electrodes comparison results.

The signal correlation coefficient between the printed and commercial electrodes was calculated using the correlation equation [31]:

$$
\text { correlation_coefficient }=\frac{\left|\sum_{i=1}^{T} y[i] * \hat{y}[i]\right|}{\sqrt{\sum_{i=1}^{T}(y[i])^{2}} \times \sqrt{\sum_{i=1}^{T}(\hat{y}[i])^{2}}}
$$

Where $\mathrm{y}$ and $\hat{\mathrm{y}}$ are the signals from the commercial electrode and the printed electrode. The correlation coefficient for the electrodes after fabrication is calculated as 0.75. However, the correlation coefficient reduced to 0.58 after experiments with participants for 30 days.

\subsection{Experiment 2: Real-time emotion detection}

In this experiment, the four printed electrodes were used to acquire the EEG signal at the locations of Fp1, Fp2, T3 and T4. T4 generates a reference signal for the differential amplifier of the two channel amplifier within the EEG-SMT and is connected to the negative terminals of both channels. Fp1 and Fp2 were connected to the positive terminals of channel one and two respectively. T3 was used to reduce the common mode interference and was connected to the driven right leg (DRL) channel which is often added to biological signal amplifiers to reduce common mode interference [32].

During the experiment, the participants were asked to wear the headband and sit in front of a computer. They were asked to keep still to reduce movement artifacts. Each participant conducted two sets of experiments: one for training the classifier and another for detection. The experimental protocol is shown in Figure 9. 


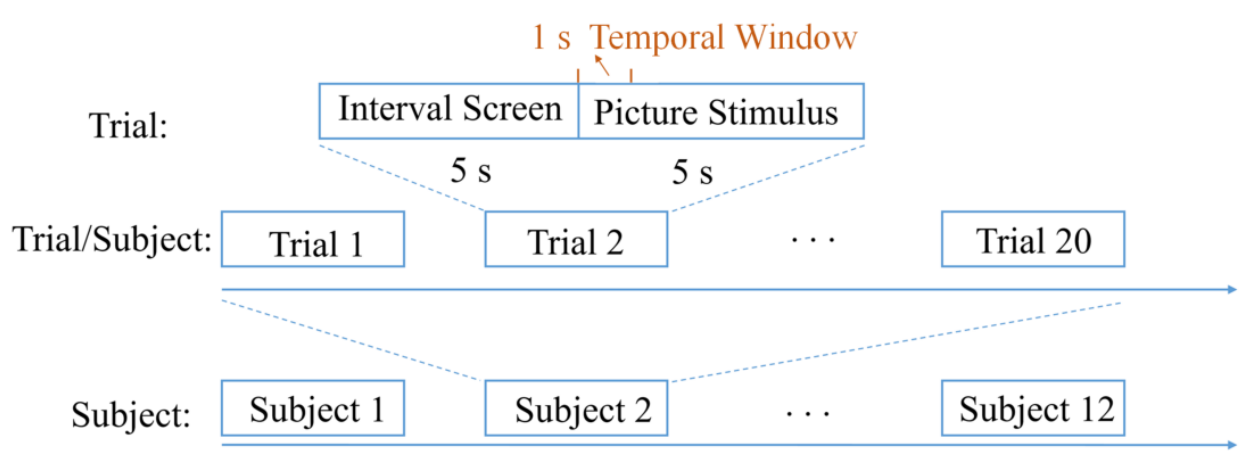

Figure 9. Experimental protocol for real-time emotion detection.

12 participants undertook the experiment with 20 trials for each participant. Each trial contained a $5 \mathrm{~s}$ interval screen and a $5 \mathrm{~s}$ picture stimulus. 20 different pictures selected from the Geneva Affective Picture Database (GAPED) [33] were randomly shown to each participant as the stimulus. Ten of the pictures were positive while the other 10 were negative. Each picture was shown to the participant for $5 \mathrm{~s}$ and the EEG signals were initially recorded for the entire $5 \mathrm{~s}$ during the stimulus. However, the emotion response only lasts for a few milliseconds [34] and it was found that only recording during the first $1 \mathrm{~s}$, after the stimulus commenced, was sufficient. Since each participant has a different latency to the stimuli, which can potentially reduce the classification accuracy, the start time of the temporal window was increased in $20 \mathrm{~ms}$ intervals until synchronisation between the recorded EEG signals and the stimulus was achieved. As a result, a $200 \mathrm{~ms}$ latency between the start of the stimuli and the temporal window occurred. We also set a further $300 \mathrm{~ms}$ delay after the "waiting time" to allow the system to execute and then show the results. Five second rest intervals occurred between each picture to allow the participants to relax and allow sufficient time to reset the emotional state [35]. After the experiment, the participants were asked to leave their feedback about the pictures, which were used to determine the labels of the stimuli.

\subsubsection{Selection of features and classification algorithms}

The EEG signals of one randomly selected participant were used to select the features and classification algorithms to provide the minimum computational time and the lowest probability of error. The EEG signal was extracted from the frequency bands of Theta, Alpha, Beta and Gamma as shown in Table 2.

Table 2. Selection of Feature and Classification algorithms.

\begin{tabular}{|l|c|c|c|}
\hline $\begin{array}{c}\text { Classification } \\
\text { Algorithms** }\end{array}$ & $\begin{array}{c}\text { LDA } \\
(\%)\end{array}$ & $\begin{array}{c}\text { SVM } \\
(\%)\end{array}$ & $\begin{array}{c}\text { MLP } \\
(\%)\end{array}$ \\
\hline Features & 54 & 42 & 51 \\
\hline Univariate statistics* & 69 & 58 & 58 \\
\hline Signal Power (SP) & 83 & 77 & 75 \\
\hline Power Spectral Density (PSD) & 63 & 59 & 61 \\
\hline Oscillation & 67 & 60 & 59 \\
\hline Common Spatial Pattern (CSP) & 88 & - & - \\
\hline CSP+SP+PSD & & & \\
\hline
\end{tabular}

* Univariate statistics include: mean, variance, range, median, Inter-Quantile-Range and Percentile of each incoming sample buffer

**Classification algorithms: Linear Discriminant Analysis (LDA), Support Vector Machine (SVM), Multilayer Perceptron (MLP).

-: Computation took several hours until system halted. 
From the table above, it can be seen that the combination of PSD, SP and CSP features achieves the highest subject-dependent accuracy when used with the Linear Discrimination Analysis (LDA) as the classification algorithm. This combination was therefore used to train the classifier.

\subsubsection{Selection of the frequency band}

The frequency band for extracting emotion features was optimised. The EEG signals from a randomly selected participant were used and the combination of PSD, SP and CSP were used as the features, with Linear Discrimination Analysis (LDA) as the classifier. The subject-dependent accuracy achieved from different frequency bands is shown in Table 3.

Table 3. Accuracy comparison between different frequency bands.

\begin{tabular}{|l|c|}
\hline Frequency band & Accuracy (\%) \\
\hline Theta & 54 \\
\hline Alpha & 68 \\
\hline Beta & 62 \\
\hline Gamma & 63 \\
\hline Theta+ Alpha+ Beta+ Gamma & 88 \\
\hline
\end{tabular}

From Table 3, it can be seen that the combination of all four frequency bands achieved the highest accuracy of $88 \%$ compared with that achieved with only a single frequency band of Theta, Alpha, Beta or Gamma.

\subsubsection{Final results and analysis}

The optimised emotion detection setup was with PSD, SP, CSP combined with LDA over four frequency bands. The results of the real-time emotion classification for 12 participants are shown in Figure 10.

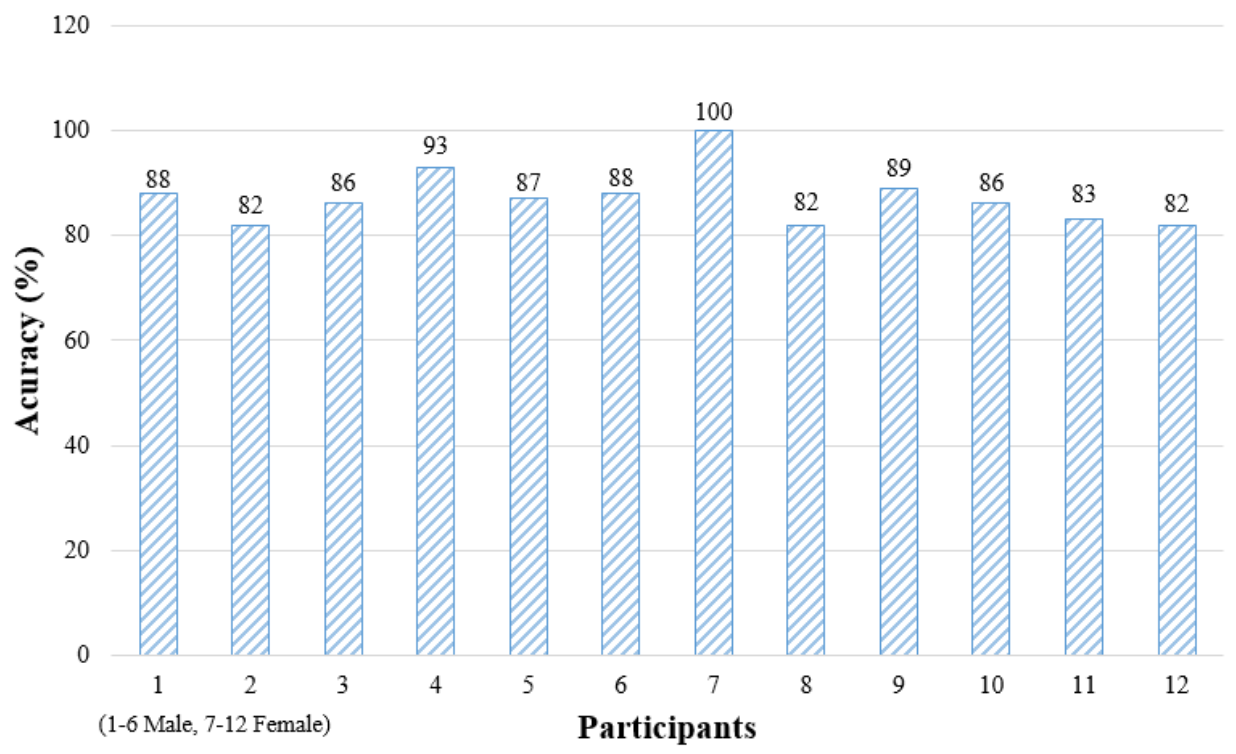

Figure 10. Real-time subject-dependent emotion classification accuracy. 
From the results, it can be observed that the accuracy of all participants is higher than $80 \%$. Further analysis shows that the standard deviation has been improved by $43 \%$ compared with previous work [24]. This is because the EEG signal was extracted from four frequency bands and that three features of the EEG signal were analysed compared with previous results, where only the alpha band and two features were used. Using three features from four frequency bands increases the information in the signal and so reduces the error.

\subsection{Experiment 3: Real-time emotion detection with an improved electrode arrangement}

The electrode arrangement reported in section 3.3 is not ideal for the vast majority of people because the location of the electrodes at $\mathrm{T} 3$ and $\mathrm{T} 4$ are on the sides of the head above the ears so that hair will reduce the signal strength and increase artifacts. This section introduces an alternative electrode arrangement, shown in Figure 11, which improves the signal strength and minimises artifacts.

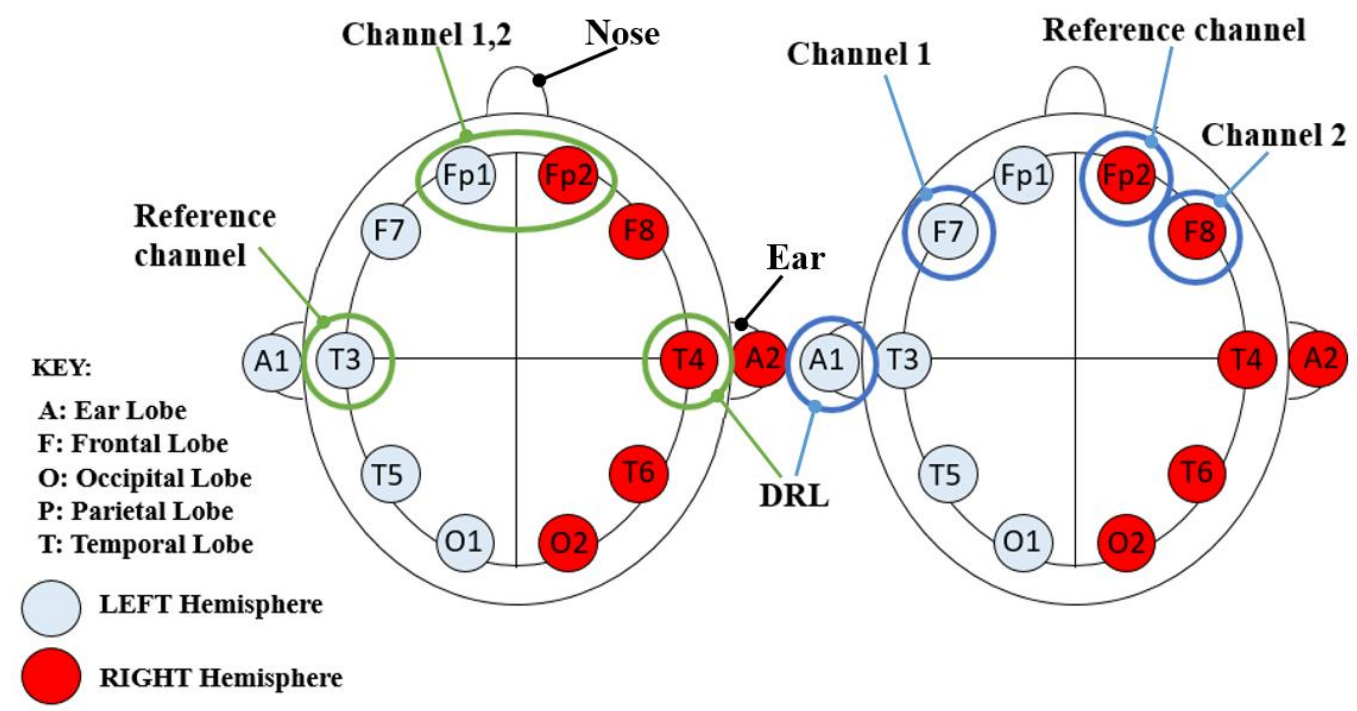

Figure 11. Top view of: (Left) Previous electrodes arrangement, (Right) optimized electrodes arrangement.

Based on the principles of attaching the electrodes to the skin as closely as possible, avoiding hair, and that the electrodes on both channels must be symmetrical, F7 and F8 were used for signal acquisition because they are located on the forehead. Fp2 was connected as a reference channel to the negative input of the differential amplifier of the two-channel EEG amplifier in the EEG-SMT. A1 was connected to a passive electrode as the driven right leg (DRL) to reduce common mode interference.

The experimental procedure, setup and stimuli were the same as described in section 3.3. The results are compared, in Error! Reference source not found., with results previously reported in the literature.

Table 4. Comparison with the real-time detection results in previous work.

\begin{tabular}{|lcccc|}
\hline & $\begin{array}{c}\text { Subject- } \\
\text { dependent } \\
\text { accuracy } \\
\text { (average) }\end{array}$ & $\begin{array}{c}\text { Standard deviation } \\
\text { (subject-dependent } \\
\text { accuracy) }\end{array}$ & $\begin{array}{c}\text { Subject- } \\
\text { independent } \\
\text { Accuracy }\end{array}$ & Real-time \\
\hline Liu et al $[21]$ & N/A & N/A & N/A & Yes \\
\hline Anh et al $[23]$ & $70.5 \%$ & N/A & N/A & Yes \\
\hline
\end{tabular}

Page 11 of 14 


\begin{tabular}{lllll}
\hline Jatupaiboon et al $[9]$ & $75.62 \%$ & N/A & $65.12 \%$ & Yes \\
\hline Matiko et al $[24]$ & $89.66 \%$ & 8.82 & $62.62 \%$ & Yes \\
\hline Present design & $86.83 \%$ & 5.03 & $64.73 \%$ & Yes \\
\hline Improved design & $91.75 \%$ & 4.57 & $66.74 \%$ & Yes \\
\hline
\end{tabular}

For the improved design, the average subject-dependant accuracy increased to $91.75 \%$ from $86.83 \%$ and the subject independent accuracy increased to $66.74 \%$ from $64.73 \%$. The standard deviation has also been further improved by $10 \%$ compared with the results presented in section 3.3. In addition, in $75 \%$ of participants, the new electrode arrangement achieved a subject-dependent accuracy higher than $90 \%$, compared with only $16 \%$ of participants in experiment 2 for the previous electrode arrangement. The emotion detection results are therefore improved because the EEG measurement took place in direct skin-contact positions hence avoiding the interference of hair.

Analysis of the results show that the average subject-dependent accuracy is lower than the prior research because of the inter participant variability. For instance, since only 12 participants are involved in this experiment, the average results can be significantly changed if the results of some participants significantly vary, which means the subject-dependent accuracy depends on the participants themselves. Therefore, the results of standard deviation and subject-independent accuracy better reflect the system performance because they evaluate the detection results of every participant rather than reflecting individual variations.

\section{Conclusions}

A real-time emotion detection system based on EEG measurement was implemented. Four screen printed active electrodes were used to acquire the EEG signals which were then processed using the open source software OpenViBE and signal processing hardware to extract features and classify the emotion state. The printing approach allows the sensing electrodes to be achieved directly on the fabric. In addition, printing enables the headband to be customised, thereby increasing the comfort of the wearer. The resistance of the printed electrodes was measured directly after fabrication at 0.25 $\Omega / \mathrm{cm}$ and the correlation coefficient compared with commercial electrodes was 0.75 . However, the resistance increased to $500 \Omega / \mathrm{cm}$ after 30 days due to contamination. Cleaning reduced the resistance to $100 \Omega / \mathrm{cm}$; the permanent change in resistance compared to the resistance when first fabricated is potentially due to mechanical failure between the electrode rubber and conductor.

From the experimental results, it is found that the combination of Power Spectral Density (PSD), Signal Power (SP) and Common Spatial Pattern (CSP) as the features achieved the highest accuracy of $86.83 \%$ when combined with the Linear Discrimination Analysis (LDA) as the classification algorithm. For the emotion detection experiments, the initial electrode arrangement at Fp1, Fp2, T3 and T4 achieves an average subject-dependent accuracy of $86.83 \%$ with a standard deviation of 5.03 and an independent accuracy of $64.73 \%$. This improvement in accuracy is because more EEG signal information was extracted than in previous research, which used fewer electrodes. However, the inter participant variability means the subject-dependent accuracy is higher than the subject-independent accuracy.

The electrode arrangement was improved by moving the electrodes to F7, F8, A1 and Fp2. This arrangement avoids the signal interference caused by hair. The subject-dependent accuracy improved to $91.75 \%$ from $86.83 \%$, which are the highest compared with other reported results using the similar setup. In addition, $75 \%$ of participants achieved a subject-dependent accuracy higher than 90 $\%$ compared with only $16 \%$ in the first design presented in experiment 2. 


\section{Acknowledgements}

The authors acknowledge the partial funding from the partnership resource of EPSRC Sphere project (http://www.irc-sphere.ac.uk ) for this work.

\section{References}

[1] R. Dolan, "Emotion, Cognition and Behavior," Science, 298, pp 1191-94, 2002.

[2] A. Bechara, "Emotion, decision making and the Orbitofrontal cortex," Cerebral Cortex, 10, pp 295-307, 2000.

[3] B. Fredrickson and T. Joiner, "Positive emotions trigger upward spirals toward emotional well-being," Psychological Science, 13, pp 172-75, 2002.

[4] E. Diener, S. Oishi and R. Lucas, "Personality, culture, and subjective well-being: Emotional and cognitive evaluations of life," Annual Review of Psychology, 54, pp 403-425, 2003.

[5] M. Rosenkranz, D. Jackson, K. Dalton, I. Dolski, C. Ryff, B. Singer, D. Muller, N. Kalin and R. Davidson, "Affective style and in vivo immune response: Neurobehavioral mechanisms," Proceedings of the National Academy of Sciences, 100, pp 11148-52, 2003.

[6] S. Cohen, W. Doyle, R. Turner, C. Alper and D. Skoner, "Emotional style and susceptibility to the common cold," Psychosomatic Medicine, 65, pp 652-57, 2003.

[7] B. Kok, K. Coffey, M. Cohn, L. Catalino, T. Vacharkulksemsuk, S. Algoe, M. Brantley and B. Fredrickson, "How positive emotions build physical health: Perceived positive social connections account for the upward spiral between positive emotions and Vagal tone," Psychological Science, 24, pp 1123-32, 2013.

[8] N. Jatupaiboon, S. Pan-ngum and P. Israsena, "Emotion classification using minimal EEG channels and frequency bands," The 2013 10th International Joint Conference on Computer Science and Software Engineering (JCSSE), Thailand, pp 21-24, 2013.

[9] N. Jatupaiboon, S. Pan-ngum and P. Israsena, "Real-time EEG-Based happiness detection system," The Scientific World Journal, 618649, pp 1-12, 2013.

[10] A. Luneski, E. Konstantinidis and P. Bamidis, "Affective medicine," Methods of Information in Medicine, 49, pp 207-218, 2010.

[11] L. Schmidt and L. Trainor, "Frontal brain electrical activity (EEG) distinguishes valence and intensity of musical emotions," Cognition and Emotion, 15, pp 487-500, 2001.

[12] L. Trainor and L. Schmidt, "Processing emotions induced by music," in I Peretz and R Zatorre, The Cognitive Neuroscience of Music, pp 310-324, 2012.

[13] N. Jatupaiboon, S. Pan-ngum and P. Israsena, "Subject-Dependent and Subject-Independent Emotion Classification Using Unimodal and Multimodal Physiological Signals," Journal of Medical Imaging and Health Informatics, 5, pp 1020-27, 2015.

[14] G. Chanel, J. Kronegg, D. Grandjean and T. Pun, "Emotion assessment: Arousal evaluation using EEG's and peripheral physiological signals," Technical report, Universite De Geneve, 2005.

[15] J. Russell, “A circumplex model of affect,” Journal of Personality and Social Psychology, 39, pp 1161-78, 1980.

[16] D. Bos, "EEG-based emotion recognition the influence of visual and Auditory stimuli," 2016. [Online]. Available: http://hmi.ewi.utwente.nl/verslagen/capita-selecta/CS-Oude_Bos-Danny.pdf. [Accessed: 11-042017]

[17] R. Levenson and H. Wagner, Emotion and the autonomic nervous system: a prospectus for research on autonomic specificity, NewYork, NY, USA: JohnWiley \& Sons, 1988, p 17-42.

[18] J. Matiko, S. Beeby and J. Tudor, "Fuzzy logic based emotion classification," 2014 IEEE International Conference on Acoustics, Speech and Signal Processing (ICASSP), Italy, 2014.

[19] F. Sharbrough, G. Chatrian, R. Lesser, H. Luders, M. Nuwer and T. Picton, "American Electroencephalographic society guidelines for standard electrode position nomenclature," Journal of Clinical Neurophysiology, 8, pp 200-202, 1991.

[20] J. Malmivuo and R. Plonsey, Bioelectromagnetism: Principles and applications of bioelectric and biomagnetic fields, New York: Oxford University Press, 1995.

[21] Y. Liu, O. Sourina and M. Nguyen, "Real-time EEG-based emotion recognition for music therapy," Journal on Multimodal User Interfaces, 5, pp 27-35, 2011. 
[22] Emotiv, [Online]. Available: https://www.emotiv.com [Accessed: 11-04-2017].

[23] V. Anh, M. Van, B. Ha and T. Quyet, "A real-time model based support vector machine for emotion recognition through EEG," 2012 International Conference on Control, Automation and Information Sciences (ICCAIS), Vietnam, 2012.

[24] J. Matiko, Y. Wei, R. Torah, N. Grabham, G. Paul, S. Beeby and J. Tudor, "Wearable EEG headband using printed electrodes and powered by energy harvesting for emotion monitoring in ambient assisted living," Smart Materials and Structures, 24, 2015.

[25] E. Yurci, "Emotion detection from EEG signals: Correlating cerebral cortex activity with musicEMOTION," 2014. [Online]. Available: http://mtg.upf.edu/node/3081 [Accessed: 11-04-2017]

[26] EEG-SMT development board USER'S MANUAL, Olimex Ltd, 2013. [Online]. Available: https://www.olimex.com/Products/EEG/OpenEEG/EEG-SMT/resources/EEG-SMT.pdf [Accessed: 11-042017].

[27] G. Paul, R. Torah, S. Beeby and J. Tunor, "Novel active electrodes for ECG monitoring on woven textiles fabricated by screen and stencil printing," Sensors and Actuators A: Physical, 221, pp 60-66, 2015.

[28] Datasheet-INA114 -Precision Instrumentation Amplifier, Texas Instruments, [Online]. Available: http://www.ti.com/product/INA114/description [Accessed: 11-04-2017]

[29] Datasheet-MCP607, Microchip, [Online]. Available: http://www.microchip.com/wwwproducts/en/MCP607 [Accessed: 11-04-2017]

[30] Datasheet-ATmega16, Atmel, [Online]. Available: http://www.atmel.com/devices/atmega16.aspx [Accessed: 11-04-2017]

[31] K. Nazarpour, H. Mohseni, C. Hesse, J. Chambers and S. Sanei, "A novel Semiblind signal extraction approach for the removal of eye-blink artifact from EEGs," EURASIP Journal on Advances in Signal Processing, 857459, pp 1-13, 2008.

[32] M. Guermandi, E. Scarselli and R. Guerrieri, "A driving right leg circuit (DgRL) for improved common mode rejection in bio-potential acquisition systems," IEEE Transactions on Biomedical Circuits and Systems, 10, pp 507-517, 2016.

[33] E. Dan-Glauser and K. Scherer, "The Geneva affective picture database (GAPED): A new 730-picture database focusing on valence and normative significance," Behavior Research Methods, 43, pp 468-77, 2011.

[34] Overview of ModularEEG, OpenEEG, [Online]. Available: http://openeeg.sourceforge.net/doc/SimpleEEG/EEGOverview.jpg [Accessed: 11-04-2017]

[35] EEG SMT schematic rev B, [Online]. Available: https://www.olimex.com/Products/EEG/OpenEEG/EEGSMT/resources/EEG-SMT-SCHEMATIC-REV-B.pdf. [Accessed: 11-04-2017]

[36] H. Aurlien, I. Gjerde, J. Aarseth, G. Eldøen, B. Karlsen, H. Skeidsvoll and N. Gilhus, "EEG background activity described by a large computerized database," Clinical Neurophysiology, 115, pp 665-73, 2004.

[37] Welcome to the OpenEEG project, [Online]. Available: http://openeeg.sourceforge.net/doc/index.html. [Accessed: 11-04-2017].

[38] S. Sanei and J. Chambers, EEG signal processing, John Wiley \& Sons Ltd, 2007.

[39] S. Cheemalapati, M. Gubanov, M. del Vale and A. Pyayt, "A real-time classification algorithm for emotion detection using portable EEG," 2013 IEEE 14th International Conference on Information Reuse \& Integration (IRI), USA, 2013.

[40] J. Matiko, S. Beeby and J. Tudor, "Real time emotion detection within a wireless sensor network and its impact on power consumption," IET Wireless Sensor Systems, 4, pp 183-90, 2014. 